\title{
Enhancement of Human Lymphocyte Transformation by Aggregated Human Gamma Globulin
}

\author{
T. Douglas Kinsella \\ From the Rheumatic Diseases Unit, Department of Medicine, Queen's \\ University, Kingston, Ontario K7L 3L6, Canada
}

\begin{abstract}
A BSTRACT The effect of heat-aggregated human gamma globulin (aggFII) on the induction of in vitro lymphocyte transformation, measured by the uptake of tritiated thymidine into newly synthesized DNA, was studied with peripheral blood lymphocytes derived from 12 patients with rheumatoid arthritis (RA), six with ankylosing spondylitis (AS), two with systemic lupus erythematosus (SLE), and seven normal subjects. It was found that $200 \mu \mathrm{g}$ aggFII induced significant transformation of the lymphocytes of eight patients with RA, five with AS, one with SLE, and one normal subject. Neither deaggregated FII nor heat-aggregated human serum albumin induced significant transformation of the lymphocytes of any subject tested. A source of complement appeared necessary to support aggFIIinduced blastogenesis, since enhanced transformation occurred only in the presence of fresh plasma. Heatinactivated plasma and fetal calf serum (FCS), and FCS devoid of hemolytic complement, failed to support enhanced blastogenesis in the presence of aggFII. Since substrates similar to those employed in these studies are present in vivo in the rheumatoid joint, it is suggested that aggFII may enhance intra-articular lymphocyte transformation in subjects with RA.
\end{abstract}

\section{INTRODUCTION}

Various in vitro studies indicate that human gamma globulin (FII), ${ }^{1}$ particularly in an aggregated form

A portion of this work appeared in abstract form in: 1972. Arthritis Rheum. 15: 444.

Dr. Kinsella's present address is the Department of Medicine, Royal Victoria Hospital, Montreal 112, Quebec, Canada.

Received for publication 26 March 1973 and in revised form 4 October 1973.

${ }^{1}$ Abbreviations used in this paper: aggFII, aggregated gamma globulin; aggHSA, aggregated human serum albumin; AS, ankylosing spondylitis; C, complement; $\mathrm{C} 3$, third component of complement; $\mathrm{C} 4$, fourth component of com-
(aggFII), can combine with rheumatoid factor (RF) to form a complex $(1,2)$ and to activate the blood complement (C) sequence $(3,4)$, thereby releasing a variety of phlogistic mediators (5). Since other studies have shown that synovial fluids derived from some patients with rheumatoid arthritis (RA) exhibit depressed levels of $\mathrm{C}(4,6)$ and can contain aggFII and $\mathrm{RF}$ (7) which is frequently in the form of intracellular immune complex-like inclusions containing $\mathrm{C}$ (8), it has been postulated that aggFII may participate in the pathogenesis of rheumatoid synovial inflammation via humoral immune mechanisms (9). Contrary to the foregoing, there has been no unequivocal demonstration that cell-mediated immunity involving FII is implicated in the pathogenesis of rheumatoicl inflammation. In particular, in vivo studies employing delayed hypersensitivity skin responses to native- and $\operatorname{agg}$ FII $(10,11)$ and the intra-articular injection of purified FII into human volunteers (12), have not resolved this question. Because of controversial results derived from such in vivo studies (10-13) others have relied on in vitro techniques, including in vitro $1 \mathrm{ym}-$ phocyte transformation which is known to be a reliable correlate of cell-mediated immunity (14), to investigate possible roles for $\mathrm{FII}$ and $\mathrm{RF}$ in the pathogenesis of RA. Unfortunately, recent studies have reported that neither isolated FII $(11,15,16)$ nor isolated RF (17) can consistently induce in vitro transformation of peripheral blood lymphocytes of subjects with RA. Thus, in contrast to the situation regarding humoral immunity, the majority of currently available evidence has not delineated a role for FII in cell-mediated immunity in the pathogenesis of RA.

plement; deaggFII, deaggregated gamma globulin; FII, human gamma globulin; FCS, fetal calf serum; HSA, human serum albumin; [3H]Tdr, tritiated thymidine; PBS, phosphate-buffered saline; PHA, phytohemagglutinin; $\mathrm{RA}$, rheumatoid arthritis; RF, rheumatoid factor; SLE, systematic lupus erythematosus. 
The present studies were suggested by observations that some circulating lymphocytes possess surface receptors for C-containing immune complexes (18) and, as reviewed above, that some RA synovial fluids contain complexes of aggFII with RF and/or C. Accordingly, the experiments to be described were designed to approximate in vivo circumstances by evaluating the blastogenic effect of aggFII and deaggregated FII (deagg FII) on peripheral blood lymphocytes in the presence of a source of $\mathrm{C}$, namely fresh autologous plasma. The results of these studies demonstrate that in vitro lymphocyte transformation can be induced by aggFII under such conditions.

\section{METHODS}

Study groups. 12 patients with RA, 6 with ankylosing spondylitis (AS), 2 with systemic lupus erythematosus (SLE), and 7 normal subjects were studied. All RA patients had "classical" or "definite" diseases by criteria of the American Rheumatism Association (19), and all but one were seropositive for $\mathrm{RF}$, as determined by a slide latex test (Hyland Div., Travenol Laboratories, Inc., Costa Mesa, Calif.) and by a micromethod for the sensitized sheep cell test, standardized with amboceptor and reference serum for RF donated by the World Health Organization (20) ; titers ranged from $1 / 8$ to $1 / 16,384$. All patients with $\mathrm{AS}$ and SLE had negative tests for RF. Active disease necessitated acetylsalicylic acid therapy in a minimum daily dose of $1,800 \mathrm{mg}$ for all patients with RA, AS, and SLE; in addition, one patient with $\mathrm{RA}$ received $10 \mathrm{mg}$ prednisone/ day, one with SLE, $250 \mathrm{mg}$ chloroquine/day, and three with AS, 200-400 mg phenylbutazone/day. None of the normal subjects displayed clinical or serological evidence of an immune disorder.

Preparation of protein solutions. Lyophilized Cohn fraction II (Grand Island Biological Co., Grand Island, N. Y.) was reconstituted in phosphate-buffered saline (PBS), $\mathrm{pH} 7.2$, to desired concentrations in volumes of $0.05 \mathrm{ml}$. Since such solutions were found to contain variable amounts of aggregates, as determined by their ability to deplete fresh plasma of hemolytic complement $(3,11)$, aliquots were deaggregated (11) by centrifugation in a Beckman model L centrifuge (Beckman Instruments, Inc., Spinco Div., Palo Alto, Calif.) at $150,000 \mathrm{~g} \times 90 \mathrm{~min}$ in a Beckman SW-50 swinging bucket rotor, and the upper one-third of the supernate (deaggFII) was sterilized by gentle passage through a $0.45-\mu \mathrm{m}$ pore Millipore filter (Millipore Corp., Bedford, Mass.). Portions of such deaggregated supernates were then heated in a water bath at $56^{\circ} \mathrm{C} \times 30 \mathrm{~min}$ to induce aggFII. Human serum albumin (HSA) (Hyland Div., Travenol Laboratories, Inc.), diluted to provide $200 \mu \mathrm{g} / 0.05 \mathrm{ml}$, was aggregated (aggHSA) by heating at $71^{\circ} \mathrm{C} \times 60 \mathrm{~min}$. Protein concentrations of aliquots of all solutions were determined, after digestion in $0.1 \mathrm{~N} \mathrm{NaOH}$ for $30 \mathrm{~min}$, in a Coleman model 111 spectrophotometer (Coleman Instruments Div., Perkin Elmer Corp., Maywood, Ill.). Protein loss during deaggregation was usually so minimal as to require no compensatory adjustment of concentration of these solutions. Studies by double diffusion in agar gel (21) and by immunoelectrophoresis (22) revealed that the solutions of FII contained predominantly $\operatorname{IgG}$ and a trace of $\operatorname{IgM}$ but no albumin, no fourth component of complement (C4), nor third component of complement (C3). Similar techniques revealed that the solutions of HSA contained only albumin and an $\alpha$-globulin which, although not further identified, was not $\operatorname{IgG}, \operatorname{Ig} \mathrm{A}, \operatorname{Ig} \mathrm{M}, \mathrm{C} 4$, nor $\mathrm{C} 3$.

Determinations. Levels of hemolytic complement $\left(\mathrm{CH}_{50}\right)$ in plasma and FII-free fetal calf serum (FCS) were determined as described by Mayer (23). The marked sensitivity of the $\mathrm{CH}_{50}$ assay to the presence of aggFII was utilized to ensure that batches of FII which had been deaggregated by centrifugation were indeed free of aggregates $(3,11)$. Plasma concentrations of C3 were determined by single radial immunodiffusion, employing commercial immunoplates (Hyland Div., Travenol Laboratories, Inc.), containing $5 \mu \mathrm{l}$ plasma/well. Studies of plasma levels of $\mathrm{C} 3$ were initiated on the day on which cultures were established for lymphocyte transformation, or, in a few instances, within $24 \mathrm{~h}$, with samples which had been immediately frozen at $-20^{\circ} \mathrm{C}$. Reaction volumes for determination of $\mathrm{C}$ activation were identical to those usually employed in lymphocyte cultures and consisted of duplicate tubes containing $0.05 \mathrm{ml}(200 \mu \mathrm{g})$ agg- or deaggFII in $0.2 \mathrm{ml}$ plasma. After incubation at $37^{\circ} \mathrm{C} \times 20 \mathrm{~min}$, appropriate aliquots were withdrawn for $\mathrm{C}$ determinations. Results are expressed as the decrease of $\mathrm{C}$ concentration in plasma after addition of $200 \mu \mathrm{g}$ aggFII as compared to the simultaneously determined $\mathrm{C}$ value in a plasma aliquot to which $200 \mu \mathrm{g}$ deaggFII had been added as a dilution control.

Leukocyte cultures. $50 \mathrm{ml}$ of venous blood, containing 1,000 units preservative-free heparin (Connaught Medical Research Laboratories, Toronto, Canada), were obtained from each subject. Erythrocytes were usually sedimented by gravity at room temperature in an inverted syringe, but for some normal subjects several centrifugations at $75 g \times 20 \mathrm{~min}$ were required. The leukocyte-rich supernatant plasma was centrifuged at $250 \mathrm{~g} \times 10 \mathrm{~min}$ in glass tubes and the resultant cell pellet, after three washes in RPMI 1640 culture medium (Grand Island Biological Co.), was resuspended in sufficient RPMI 1640 to provide $5 \times$ $10^{5}$ small lymphocytes in $0.2 \mathrm{ml}$. Supernatant plasma was centrifuged once again at $1500 \mathrm{~g} \times 10 \mathrm{~min}$ at $4^{\circ} \mathrm{C}$ before addition to autologous cultures and, when required in some experiments, heat inactivated at $56^{\circ} \mathrm{C} \times 30 \mathrm{~min}$ in a water bath. FII-free FCS (Grand Island Biological Co.) was employed in the fresh and/or heat-inactivated form in some experiments.

Cultures were established in triplicate or quadruplicate in final volumes of $1.0 \mathrm{ml}$, containing $5 \times 10^{5}$ lymphocytes, $20 \%$ autologous plasma or FCS, $0.05 \mathrm{ml} \mathrm{FII,} \mathrm{and} 0.5 \%$ of $200 \mathrm{mM} l$-glutamine and antibiotics. Initial experiments employing concentrations of $50,100,200,600$, and 1,000 $\mu \mathrm{g}$ of agg- and deaggFII in $0.05-\mathrm{ml}$ volumes indicated that $200 \mu \mathrm{g}$ usually provided optimal in vitro mitogenic responses and, accordingly, all experiments reported herein were conducted in the presence of $200 \mu \mathrm{g}$ FII (agg or deagg)/culture tube. In the majority of experiments, additional cultures containing fresh autologous plasma and 0.05 $\mathrm{ml}$ of a $1 / 10$ dilution of phytohemagglutinin (PHA) (Difco Laboratories, Detroit, Mich.), added on the 3rd day of incubation, were established to ensure that cultured cells were capable of undergoing a mitogenic response. In some experiments, additional cultures were established containing $200 \mu \mathrm{g}$ aggHSA to evaluate the magnitude of tritiated thymidine $\left(\left[{ }^{3} \mathrm{H}\right] \mathrm{Tdr}\right)$ uptake by proliferation of phagocytic leukocytes during culture. 
TABLE I

Summary of Lymphocyte Transformation Responses to PHA, deaggFII, and aggFII Correlated with Original C3 Levels and Reduction Induced by aggFII

\begin{tabular}{|c|c|c|c|c|c|c|}
\hline \multirow{3}{*}{$\begin{array}{l}\text { Lymphocyte } \\
\text { donor }\end{array}$} & \multirow[b]{3}{*}{ Control cultures } & \multirow{2}{*}{\multicolumn{3}{|c|}{ MI\& in presence of }} & \multicolumn{2}{|c|}{ C.3 } \\
\hline & & & & & \multirow{2}{*}{$\begin{array}{l}\text { Native } \\
\text { plasma }\end{array}$} & \multirow{2}{*}{$\begin{array}{l}\text { Reduction } \\
\text { by aggFII }\end{array}$} \\
\hline & & PHA & deaggFII\| & $\operatorname{aggFIIq~}$ & & \\
\hline \multicolumn{7}{|l|}{ RA } \\
\hline T. W. & $107 \pm 8$ & 37.4 & 1.4 & 7.6 & 1.35 & 0.27 \\
\hline D. L. & $266 \pm 108$ & 403.5 & _** & 4.7 & 1.78 & 0.81 \\
\hline C. C. & $407 \pm 66$ & 2.5 & - & 4.6 & 2.30 & 0.75 \\
\hline J. Pl. & $285 \pm 43$ & 2.4 & 1.3 & 3.2 & 1.00 & 0.26 \\
\hline C. S. & $250 \pm 115$ & 13.1 & $1.7 \ddagger$ & 2.9 & 0.89 & 0.08 \\
\hline A. L. & $1,243 \pm 325$ & 27.3 & 1.4 & 2.7 & 0.90 & 0.11 \\
\hline J. F. & $127 \pm 81$ & 159.1 & $2.4 \ddagger \ddagger$ & 2.2 & 0.94 & 0.24 \\
\hline J. P. & $674 \pm 167$ & 9.4 & - & 2.1 & 1.32 & 0.17 \\
\hline E. R. & $167 \pm 17$ & 3.0 & 0.8 & 1.8 & 1.06 & 0.18 \\
\hline C. L. & $327 \pm 66$ & 16.2 & 0.7 & 1.6 & 1.80 & 0.00 \\
\hline E. E. & $347 \pm 30$ & 53.7 & - & 1.1 & 1.60 & 0.05 \\
\hline E. C. & $339 \pm 50$ & 68.4 & 1.2 & 1.0 & 0.97 & 0.12 \\
\hline Mean $( \pm S E)$ & $385( \pm 88)$ & $66.3( \pm 33.2)$ & $1.3( \pm 0.4)$ & $2.9( \pm 0.5)$ & $1.32( \pm 0.13)$ & $0.25( \pm 0.07)$ \\
\hline \multicolumn{7}{|c|}{ (6) } \\
\hline G. C. & $1,887 \pm 432$ & 72.1 & - & 9.5 & 1.75 & 0.25 \\
\hline A. $\mathrm{R}$. & $1,092 \pm 90$ & 48.0 & - & 8.6 & 1.40 & 0.41 \\
\hline H. M. & $323 \pm 40$ & 245.8 & - & 5.8 & 1.29 & 0.43 \\
\hline J. L. & $608 \pm 3$ & 116.5 & - & 2.9 & 1.33 & 0.39 \\
\hline I. F. & $280 \pm 31$ & 210.4 & - & 2.2 & 1.55 & 0.26 \\
\hline E. W. & $180 \pm 27$ & 67.1 & 0.9 & 1.4 & 2.75 & 0.45 \\
\hline Mean $( \pm \mathrm{SE})$ & $728( \pm 268)$ & $126.6( \pm 33.6)$ & - & $5.0( \pm 1.4)$ & $1.67( \pm 0.22)$ & $0.36( \pm 0.03)$ \\
\hline \multicolumn{7}{|l|}{ SLE } \\
\hline J. A. & $426 \pm 103$ & 78.4 & 1.4 & 3.0 & 0.86 & 0.07 \\
\hline L. T. & $390 \pm 66$ & 116.9 & 0.6 & 1.5 & 0.57 & 0.07 \\
\hline \multicolumn{7}{|l|}{ Normal } \\
\hline P. W. & $301 \pm 48$ & 142.2 & 0.9 & 4.4 & 1.20 & 0.15 \\
\hline I. P. & $819 \pm 34$ & 13.3 & - & 1.6 & 1.90 & 0.15 \\
\hline A. C. & $376 \pm 188$ & 57.2 & 1.4 & 1.6 & 1.17 & 0.15 \\
\hline J. N. & $2,507 \pm 981$ & 4.3 & - & 1.5 & 2.00 & 0.20 \\
\hline A. B. & $249 \pm 58$ & 6.6 & - & 0.9 & 1.41 & 0.44 \\
\hline F. W. & $941 \pm 245$ & 35.7 & - & 0.9 & 1.17 & 0.23 \\
\hline J. D. & $140 \pm 23$ & - & 0.7 & 0.9 & - & - \\
\hline Mean $( \pm S E)$ & $761( \pm 312)$ & $43.2( \pm 21.4)$ & $1.0( \pm 0.2)$ & $1.6( \pm 0.4)$ & $1.47( \pm 0.15)$ & $0.22( \pm 0.04)$ \\
\hline
\end{tabular}

* Control cultures, $\mathrm{cpm}=$ counts per minute in unstimulated control cultures.

$\ddagger \mathrm{PHA}=0.05 \mathrm{ml}$ PHA added on 3rd day of culture.

$\S \mathrm{MI}=$ mean counts per minute in experimental cultures divided by mean counts per minute in control cultures; all cultures contain fresh autologous plasma and were harvested after $5 \frac{1}{2}$ days.

$\|$ deaggFII $=200 \mu \mathrm{g}$ FII, deaggregated by centrifugation at 150,000 $\mathrm{g} \times 90 \mathrm{~min}$.

I aggFII $=200 \mu \mathrm{g}$ FII, aggregated by heating $\left(56^{\circ} \mathrm{C} \times 30 \mathrm{~min}\right)$.

** $(-)=$ not done.

$\ddagger \ddagger$ Preparations of deaggFII employed in these experiments decreased the titer of hemolytic complement in fresh plasma by more than $25 \%$; all other, less than $10 \%$.

Cultures were maintained in loosely capped polypropylene in air and were pulsed during the final $18 \mathrm{~h}$ with $2.0 \mu \mathrm{Ci}$ tubes (Falcon Plastics, Div. of BioQuest, Oxnard, Calif.) of $\left[{ }^{3} \mathrm{H}\right] \mathrm{Tdr}$, sp act $5.0 \mathrm{Ci} / \mathrm{mmol}$ (Amersham/Searle Corp., for $5 \frac{1}{2}$ days at $37^{\circ} \mathrm{C}$ in a humidified atmosphere of $5 \% \mathrm{CO}_{2}$ Arlington Heights, Ill.). They were then processed for 
liquid scintillation counting as reported previously (24) and counted in a Philips liquid scintillation counter (Philips Electronic Instruments, Mount Vernon, N. Y.), employing an external standard and a correction for background counts. In some experiments identical cultures were established and processed for liquid scintillation counting at both 3 days and $5 \frac{1}{2}$ days to evaluate the effect of duration of culture on aggFII-induced blastogenesis. Results of all experiments are expressed as a mitogenic index (MI) which is the ratio obtained by dividing mean counts per minute in experimental cultures (containing agg- or deaggFII) by those in control cultures which contained no FII. MI of 2.0 or greater were arbitrarily chosen to represent enhanced lymphocyte transformation in this system. A Student's $t$ test, employing an Olivetti Programma 101 calculator (Olivetti Underwood Corp., New York), was applied to evaluate the significance of the difference between the means of each of the study groups.

\section{RESULTS}

Blastogenic effects of aggFII and PHA. MI for each subject in response to $200 \mu \mathrm{g}$ aggFII or $0.05 \mathrm{ml}$ PHA in fresh plasma are shown in Table I. MI in response to aggFII for the $12 \mathrm{RA}$ subjects ranged from 1.0 to 7.6 with a mean of 2.9 ( $\mathrm{SE} \pm 0.5$ ), while those for the normal group ranged from 0.9 to 4.4 with a mean of $1.6(\mathrm{SE} \pm 0.4) \quad(P>0.05$ in comparison to the normal group). The range of MI for the six AS subjects was 1.4-9.5 with a mean of 5.0 (SE $\pm 1.4)(P<0.05$ in comparison to the normal group). The two SLE subjects had MI of 3.0 and 1.5. In total, 15 of the 27 subjects had MI of 2.0 or greater in response to aggFII, mean 4.4 ( $\mathrm{SE} \pm 0.6$ ), while 12 subjects had MI less than 2.0, mean $1.2(\mathrm{SE} \pm 0.1)$; the difference between these means was significant $(P<0.001)$.

In 3-day cultures (data not shown) aggFII failed to induce significant transformation of lymphocytes from three subjects with RA, one each with $A S$ and SLE, and two normal subjects.

MI after PHA stimulation are recorded (Table I, column 2) for all but one subject (normal, J. D.). No correlation was observed between the magnitude of aggFII and PHA MI for individual subjects (Table I, columns 2 and 4 ).

Blastogenic effect of aggFII compared to deaggFII. Comparative MI for aggFII and deaggFII in parallel cultures containing fresh autologous plasma can be seen in Table I (columns 3 and 4). The range of MI for eight RA subjects was $1.0-7.6$ in the presence of aggFII and 0.7-2.4 in the presence of deaggFII. In the latter experiments, MI of 1.7 and 2.4 in the presence of deaggFII were encountered with two subjects; the preparations of deaggFII employed in these two experiments were found to consume more than $25 \%$ $\mathrm{CH}_{\text {so }}$ units in native autologous plasma, thereby suggesting inadequate deaggregation of the FII. One AS
TABLE II

Comparison of Lymphocyte Transformation Responses to aggFII and agg HSA

\begin{tabular}{|c|c|c|c|}
\hline \multirow{2}{*}{$\begin{array}{l}\text { Lymphocyte } \\
\text { donor }\end{array}$} & \multirow[b]{2}{*}{ Diagnosis } & \multicolumn{2}{|c|}{$M I^{*}$ in presence of } \\
\hline & & aggFII & aggHSA\& \\
\hline T. W. & RA & 7.6 & 1.7 \\
\hline C. S. & $\mathrm{RA}$ & 2.9 & 0.6 \\
\hline A. L. & RA & 2.7 & 1.3 \\
\hline C. L. & RA & 1.6 & 0.9 \\
\hline L. T. & SLE & 1.5 & 1.1 \\
\hline E. W. & AS & 1.4 & 1.0 \\
\hline \multicolumn{2}{|c|}{ Mean ( $(\mathrm{SE})$} & $2.9( \pm 0.9)$ & $1.1( \pm 0.1)$ \\
\hline
\end{tabular}

* MI = mean counts per minute in experimental cultures divided by mean counts per minute in control cultures; all cultures contain fresh autologous plasma.

$\ddagger$ aggFII $=200 \mu \mathrm{g}$ heat aggregated $\left(56^{\circ} \mathrm{C} \times 30 \mathrm{~min}\right) \mathrm{FII}$.

$\S$ aggHSA $=200 \mu \mathrm{g}$ heat aggregated $\left(71^{\circ} \mathrm{C} \times 60 \mathrm{~min}\right) \mathrm{HSA}$.

subject had MI of 1.4 and 0.9 in the presence of aggFII and deaggFII, respectively. Two SLE subjects had MI of 3.0 and 1.5 with aggFII, and 1.4 and 0.6 in the presence of deaggFII. Three normal subjects had a range of MI from 0.9 to 4.4 in the presence of aggFII and from 0.7 to 1.4 in the presence of deaggFII. These studies demonstrated that $200 \mu \mathrm{g}$ deaggFII was unable to induce blastogenesis of peripheral blood lymphocytes in the presence of fresh plasma.

Effect of aggHSA on uptake of $\left[{ }^{3} H\right] T d r$. To ascertain the magnitude of $\left[{ }^{8} \mathrm{H}\right] \mathrm{Tdr}$ uptake due to synthesis of DNA by phagocytic leukocytes in 5-day cultures, the leukocytes of four subjects with RA and one each with SLE and AS were cultured in parallel in the presence of $200 \mu \mathrm{g}$ each of aggFII and aggHSA (Table II). In the presence of aggFII three of six subjects had MI greater than 2.0, whereas in the presence of aggHSA all had MI less than 2.0, indicating that in this system an MI of 2.0 or greater was unlikely to represent uptake of $\left[{ }^{3} \mathrm{H}\right] \mathrm{Tdr}$ by in vitro proliferation of phagocytic leukocytes.

Effect of inactivation of $C$ on blastogenic responses. In seven experiments (Table III, columns 1 and 2) $200 \mu \mathrm{g}$ aggFII was added to parallel cultures containing fresh plasma or plasma which had been decomplemented by heat inactivation before addition of aggFII. Heat inactivation inhibited the blastogenic response to aggFII which the lymphocytes of one subject with RA $(\mathrm{MI}=3.2)$ and two with AS (MI = 5.8 and 2.2) had displayed in the presence of fresh plasma. After heat inactivation the decrease in $\mathrm{CH}_{50}$ units in plasmas ranged from $91 \%$ to $99 \%$ with a mean decrease of $94 \%$.

MI obtained in response to $200 \mu \mathrm{g}$ aggFII in autolo- 
TABLE III

Comparison of Lymphocyte Transformation Responses in Autologous Plasma and FCS

\begin{tabular}{|c|c|c|c|c|}
\hline \multirow{3}{*}{$\begin{array}{c}\text { Lymphocyte } \\
\text { donor }\end{array}$} & \multicolumn{4}{|c|}{ MI* with aggFII $\ddagger$ in } \\
\hline & \multicolumn{2}{|c|}{ Autologous plasma } & \multicolumn{2}{|c|}{ Fetal calf serum } \\
\hline & Fresh§ & Inactivated $\|$ & Fresh $\Phi$ & Inactivated $\|$ \\
\hline \multicolumn{5}{|l|}{ RA } \\
\hline T. W. & 7.6 & $\ldots * *$ & 1.2 & 0.6 \\
\hline J. Pl. & 3.2 & 0.8 & - & - \\
\hline J. P. & 2.1 & - & - & 1.1 \\
\hline E. E. & 1.1 & 0.5 & 0.9 & 1.2 \\
\hline E. C. & 1.0 & - & - & 0.8 \\
\hline \multicolumn{5}{|l|}{ AS } \\
\hline J. M. & 5.8 & 0.7 & - & - \\
\hline I. F. & 2.2 & 1.1 & - & 0.8 \\
\hline E. W. & 1.4 & $\cdots$ & 0.7 & 0.9 \\
\hline \multicolumn{5}{|l|}{ Normal } \\
\hline A. C. & 1.6 & 1.4 & 1.1 & 0.7 \\
\hline J. N. & 1.5 & 1.0 & - & - \\
\hline J. D. & 0.9 & - & 1.0 & - \\
\hline F. W. & 0.9 & 0.9 & $\ldots$ & - \\
\hline
\end{tabular}

* MI = counts per minute in cultures containing aggFII divided by mean counts per minute in cultures without aggFII.

$\ddagger$ aggFII $=200 \mu \mathrm{g}$ heat-aggregated $\left(56^{\circ} \mathrm{C} \times 30 \mathrm{~min}\right)$ human gamma globulin.

$\S$ Fresh = autologous plasma obtained on day on which cultures established. || Inactivated = autologous plasma or commercial calf serum heated in a water bath at $56^{\circ} \mathrm{C} \times 30 \mathrm{~min}$ to inactivate hemolytic complement.

If Fresh = fetal calf serum obtained frozen from commercial supplier; she lf life greater than five days.

$* *(-)=$ not done.

gous plasma or in FCS are also compared in Table III. Moderate to marked enhancement of lymphocyte transformation was displayed by two subjects with RA ( $\mathrm{MI}=7.6$ and 2.1) and one with $\mathrm{AS}(\mathrm{MI}=2.2)$ in the presence of fresh autologous plasma (Table III, column 1), but no enhancement occurred with any subject in parallel cultures containing heat-inactivated FCS (Table III, column 4). In some of these experiments the mitogenicity of aggFII was also compared in parallel cultures of both fresh and heat-inactivated plasma and FCS (Table III). It was found that only fresh autologous plasma supported aggFII-induced blastogenesis; neither fresh nor heat-inactivated FCS supported a blastogenic response to aggFII (Table III, columns 3 and 4). Aliquots of three separate batches of FCS were employed in these experiments, and none were found to contain detectable hemolytic complement activity in either the fresh or heat-inactivated preparations. It should be noted that although the "fresh" preparations of FCS were received frozen from a commercial supplied they had an undetermined "shelf life" before delivery. These experiments indicated that under otherwise identical experimental conditions a source of $\mathrm{C}$ was required for expression of aggFIIinduced blastogenesis.
Effect of aggFII on plasma C3 levels. Except for one normal subject, C3 concentrations were determined before and after the addition of $200 \mu \mathrm{g}$ aggFII to plasma in all experiments (Table I, columns 5 and 6). For individual subjects and diagnostic groups no correlation was found between the degree of blastogenesis and the extent of $\mathrm{C} 3$ depletion by aggFII (Table I, columns 4 and 6 ). When the responses of the 15 subjects with enhanced blastogenesis (MI > 2.0) were compared to those of 11 subjects without such enhancement, it was found that the mean decrease in the level of plasma C3 after addition of aggFII was $0.31 \mathrm{mg} / \mathrm{ml}$ (range $0.07-0.81 \mathrm{mg} / \mathrm{ml}$ ) for the former group and $0.18 \mathrm{mg} / \mathrm{ml}$ (range $0-0.45 \mathrm{mg} / \mathrm{ml}$ ) for the latter $(P<0.1)$.

\section{DISCUSSION}

The present studies demonstrate that the addition of $200 \mu \mathrm{g}$ of aggFII, but not deaggFII to fresh autologous plasma was associated with enhanced in vitro blastogenic transformation of the peripheral blood lymphocytes of 15 of 27 subjects. Specifically, the mean response of the 15 subjects with MI of 2.0 or greater was 4.4 , in comparison to a mean of 1.2 for the 12 subjects with indices of less than 2.0; the difference between these means was highly significant statistically $(P<0.001)$. In addition, by employing the same MI of 2.0 or greater to define enhanced blastogenesis, it was found that aggFII induced such enhancement with lymphocytes derived from 8 of 12 patients with RA, 5 of 6 with AS, 1 of 2 with SLE, and 1 of 7 normal subjects. Thus, the results of these studies indicate that in the presence of fresh autologous plasma, aggFII can induce significant transformation of the blood lymphocytes of some normal individuals and patients with immune arthropathies.

No statistically significant differences between the mean responses of the 7 normal subjects (mean $\mathrm{MI}=$ $1.6)$, the 12 patients with RA (mean $\mathrm{MI}=2.9$ ), and 2 patients with SLE (MI $=3.0$ and 1.5) were found. Although lymphocytes of the AS patients did demonstrate a quantitatively greater mean response than those of the other study groups, other studies conducted in this laboratory and reported in abstract form indicate that AS lymphocytes, in comparison to normal and RA lymphocytes, also display greater in vitro blastogenesis in the mixed leukocyte reaction and in response to PHA (25). Thus, the results of the present studies with aggFII provide additional evidence for an increased sensitivity of AS lymphocytes to a variety of blastogenic stimuli. In the context of the present studies, therefore, it would appear that aggFII-induced blastogenesis is not specific for lymphocytes derived from subjects with immune arthropathies, although 
lymphocytes of the latter subjects respond to a fixed dose of aggFII more frequently than do those of normal subjects.

Since the present studies were designed to approximate the intra-articular cellular milieu of RA, a mixed population of peripheral blood leukocytes was employed in culture. However, such a leukocyte population contains mononuclear phagocytes (monocytes) which can proliferate in vivo by mitotic division in response to inflammatory phagocytic stimuli (26). Accordingly, in the present experiments it was necessary to differentiate in vitro DNA synthesis $\left(\left[{ }^{3} \mathrm{H}\right] \mathrm{Tdr}\right.$ uptake) which might be due to mitosis of phagocytic monocytes and/ or macrophages from that which was due to mitosis of co-cultivated lymphocytes. Since it is known that mononuclear phagocytes avidly phagocytose aggregated HSA (27), experiments were designed to compare $\left[{ }^{3} \mathrm{H}\right] \mathrm{Tdr}$ uptake in parallel cultures containing aggHSA and aggFII. These experiments demonstrated that the uptake of $\left[{ }^{3} \mathrm{H}\right] \mathrm{Tdr}$, expressed as an $\mathrm{MI}$, was invariably greater in the presence of aggFII than in the presence of a similar amount of aggHSA, thereby indicating that the observed $\left[{ }^{3} \mathrm{H}\right] \mathrm{Tdr}$ uptake was not solely a reflection of macrophage proliferation in response to a phagocytic stimulus.

Current evidence indicates that human peripheral blood contains at least two populations of morphologically similar, but functionally distinct, lymphocytes, namely, thymus-derived $\mathrm{T}$ cells and bone marrow-derived $B$ cells (reviewed in reference 28 ). In addition, it has been shown that $\mathrm{T}$ cells, but not $\mathrm{B}$ cells, undergo in vitro blastogenic transformation in response to $\mathrm{PHA}$ (29) and that $B$ cells possess surface membrane receptors for $\mathrm{C}$ (18) and for immunoglobulins, including aggFII (30). In view of these considerations, the results of the present studies suggest that the observed response to aggFII may have been mediated by $B$ cells. In particular, the lack of correlation in the present studies between PHA and aggFII blastogenic responses for individual subjects would support such an interpretation, as would those studies which have demonstrated that $B$ cells possess surface receptors for complement-fixing immune complexes (18) and for aggFII (30). Thus, with respect to the present studies, it is attractive to speculate that complexes formed in vitro between aggFII with $R F$ and/or $C$ reacted with $\mathrm{B}$ cells bearing appropriate receptors and initiated the observed blastogenic sequence. Studies have been commenced which will directly evaluate this possibility.

The failure of deaggFII, which does not fix C (3), to induce blastogenesis in the presence of fresh autologous plasma suggests that participation of $\mathrm{C}$ may have been necessary for expression of the reaction described. This interpretation is augmented by those experiments in which it was demonstrated that both heat-inactivated plasma and FCS devoid of hemolytic complement were also unable to support aggFII-induced blastogenesis. Indeed, this requirement for fresh plasma may account for the negative results reported by previous investigators who employed heat-inactivated human AB sera (15) or calf sera (16) in similar studies with human FII. At the same time it is clear that activation of the $C$ sequence by aggFII was not the sole requisite for expression of this reaction; although the mean depletion of plasma C3 by aggFII in the group of 15 subjects with enhanced blastogenesis was greater than that in the group of 12 subjects without enhanced blastogenesis, individual subjects demonstrated no significant quantitative correlations between depletion of plasma C3 and enhancement of lymphocyte blastogenesis. Thus, the foregoing considerations suggest that several factors may regulate the extent of aggFII-induced blastogenesis, namely, activation of the $C$ system, an appropriate amount of aggFII, and the presence of an appropriate number of B cells. Indeed, this interpretation is consistent with a recent hypothesis which suggests that activated $C 3$ is required for $B$ cell activation (31) and with current studies conducted by the author which demonstrate that, in fresh autologous plasma, activation of the alternate pathway of $\mathrm{C}$ by endotoxin, zymosan, and inulin initiates dose-dependent levels of in vitro blastogenesis of human lymphocytes in the absence of any mitogen ( $T$. D. Kinsella, unpublished observations).

In conclusion, the present studies suggest a mechanism whereby aggFII might assist in perpetuating the subsynovial lymphoid hyperplasia which is so characteristic of some inmune arthropathies, such as RA. Various authors have demonstrated that aggFII is present in rheumatoid synovial fluids (7), that activation and utilization of $\mathrm{C}$ occurs within the RA joint $(4,6)$, and that intra-articular complexes of IgG and $C$ are present in patients with RA (8). Accordingly, although comparable information is not available for other immune arthropathies, studies of the intra-articular milieu in patients with RA have provided abundant evidence of the in vivo availability of substrates similar to those utilized in the present in vitro studies. By analogy with the present studies, therefore, the availability of C-fixing aggregated $\operatorname{IgG}$ in the $\mathrm{RA}$ joint might provoke in vivo intrasynovial lymphocyte transformation and persistent subsynovial lymphoid hyperplasia. Support for this concept can be derived from other studies conducted in this laboratory which have demonstrated that the majority of RA synovial fluids are mitogenic in vitro for autologous lymphocytes (24). Although it is likely that the reaction described in the present studies is but a secondary phe- 
nomenon, and not the primary inducer of RA (or immune) synovitis, it is apparent that such a mechanism could contribute to the propogation of chronic rheumatoid synovitis, with or without persistence of the primary inducer. In effect, these studies suggest that aggFII could support rheumatoid synovitis via cellular as well as humoral immune mechanisms.

Addendum. After this work was submitted, a paper by M. D. Reynolds and N. I. Abdou was published (1973. J. Clin. Invest. 52: 1627), which confirmed that aggregated gamma globulin can induce in vitro transformation of human lymphocytes.

\section{ACKNOWLEDGMENTS}

The technical assistance of Liliane LeBrun, Ann Sayer, and Susan Sheldon is gratefully acknowledged, as is the secretarial assistance of Frances Gareau and Terry Kelly. These studies were supported by a grant from the Canadian Arthritis and Rheumatism Society (6-155-71).

\section{REFER ENCES}

1. Kunkel, H. G., and E. M. Tan. 1964. Autoantibodies and disease. Adv. Immunol. $4: 351$.

2. Bozoky, S., and E. C. Franklin. 1966. Effects of chemical modifications of human $\gamma$-globulins on their interaction with rheumatoid factor and antibodies. Arthritis Rheum. 9: 405.

3. Christian, C. L. 1960. Studies of aggregated $\gamma$-globulin. I. Sedimentation, electrophoretic and anticomplementary properties. J. Immunol. 84: 112.

4. Zvaifler, N. J. 1969. Breakdown products of $\mathrm{C}^{\prime} 3$ in human synovial fluids. J. Clin. Invest. 48: 1532.

5. Ruddy, S., I. Gigli, and K. F. Austen. 1972. The complement system of man. N. Engl. J. Med. 287: 489.

6. Townes, A. S., and R. L. Marcus. 1972. Complementfixing activity of rheumatoid synovial fluid. Ann. Rheum. Dis. $31: 393$.

7. Hannestad, K. 1967. Presence of aggregated $\gamma$ G-globulin in certain rheumatoid synovial effusions. Clin. Exp. Immunol. 2: 571 .

8. Kinsella, T. D., J. Baum, and M. Ziff. 1969. Immunofluorescent demonstration of an IgG- $\beta_{1 c}$ complex in synovial lining cells of rheumatoid synovial membrane. Clin. Exp. Immunol. 4: 265.

9. Hollander, J. L., D. J. McCarty, Jr., G. Astorga, and E. Castro-Murillo. 1965. Studies on the pathogenesis of rheumatoid joint inflammation. I. The "R. A. cell" and a working hypothesis. Ann. Intern. Med. 62: 271.

10. Chamberlain, M. A., C. G. Shapland, and I. M. Roitt. 1970. Response to autologous immunoglobulin $G$ in patients with rheumatoid arthritis. Ann. Rheum. Dis. $29: 173$.

11. Runge, L. A., and J. A. Mills. 1971. In vivo and in vitro responses to autologous IgG in patients with rheumatoid arthritis. Arthritis Rheum. 14: 631.

12. Restifo, R. A., A. J. Lussier, A. J. Rawson, J. H. Rockey, and J. L. Hollander. 1965. Studies on the pathogenesis of rheumatoid joint inflammation. III. The experimental production of arthritis by the intra-articular injection of purified 7S gamma globulin. Ann. Intern. Med. $62: 285$.
13. Sliwinski, A. J., and N. J. Zvaifler. 1969. The removal of aggregated and nonaggregated autologous gamma globulin from rheumatoid joints. Arthritis Rheum. 12: 504 .

14. Valentine, F. 1971. Lymphocyte transformation. The proliferation of human blood lymphocytes stimulated by antigen in vitro. In Vitro Methods in Cell-Mediated Immunity. B. R. Bloom and P. R. Glade, editors. Academic Press, Inc., New York. 443.

15. Moller, G. 1969. Induction of DNA synthesis in normal human lymphocyte cultures by antigen-antibody complexes. Clin. Exp. Immunol. $4: 65$.

16. Kacaki, J. N., W. E. Bullock, and J. H. Vaughan. 1969. Failure of lymphocyte transformation in response to gamma globulins in rheumatoid arthritis. Lancet. 1 : 1289.

17. King, R. A., R. P. Messner, and R. C. Williams. 1969. Human lymphocyte transformation induced by anti-gamma globulin factors. Arthritis Rheum. 12: 597.

18. Bianco, C., R. Patrick, and V. Nussenzweig. 1970. A population of lymphocytes bearing a membrane receptor for antigen-antibody-complement complexes. I. Separation and characterization. J. Exp. Med. 132: 702.

19. Ropes, M., G. A. Bennett, S. Cobb, R. Jacox, and R. Jessar. 1958. 1958 revision of diagnostic criteria for rheumatoid arthritis. Bull. Rheum. Dis. 9: 175.

20. Anderson, S. G., M. W. Bentzon, V. Houba, and P. Krag. 1970. International reference preparation of rheumatoid arthritis serum. Bull. W. H. O. 42: 311.

21. Ouchterlony, O. 1962. Diffusion-in-gel methods for immunological analysis. Prog. Allergy. 6: 30.

22. Scheidegger, J. J. 1955. Une micro-méthode de l'inmunoeléctrophorèse. Int. Arch. Allergy Appl. Immunol. $7: 103$.

23. Mayer, M. M. 1971. Complement and complement fixation. In Experimental Immunochemistry. E. A. Kabat and M. M. Mayer, editors. Charles C Thomas, Publisher, Springfield, Ill. 2nd edition. 133.

24. Kinsella, T. D. 1973. Induction of autologous lymphocyte transformation by synovial fluid from patients with rheumatoid arthritis. Clin. Exp. Immunol. 14: 187.

25. Kinsella, T. D. 1972. Studies of selected parameters of cellular and humoral immunity in patients with ankylosing spondylitis. Ann. R. Coll. Physicians Surg. Can. 5: 38. (Abstr.)

26. Spector, W. G., and G. B. Ryan. 1970. The mononuclear phagocyte in inflammation. In Mononuclear Phagocytes, R. van Furth, editor. Blackwell Scientific Publications Ltd., Oxford. 15: 219.

27. Lang, P. G., and G. L. Ada. 1967. The localization of heat denatured serum albumin in rat lymph nodes. Aust. J. Exp. Biol. Med. Sci. 45: 445.

28. Craddock, C. G., R. Longmire, and R. McMillan. 1971. Lymphocytes and the immune response. N. Engl. J. Med. 258: 324.

29. Janossy, G., and M. F. Greaves. 1971. Lymphocyte activation. I. Response of $\mathrm{T}$ and $\mathrm{B}$ lymphocytes to phytomitogens. Clin. Exp. Immunol. 9: 483.

30. Dickler, H. B., and H. G. Kunkel. 1972. Interaction of aggregated $\gamma$-globulin with B lymphocytes. J. Exp. Med. 136: 191.

31. Dukor, P., and K. U. Hartmann. 1973. Hypothesis. Bound $\mathrm{C} 3$ as the second signal for $\mathrm{B}$ cell activation. Cell. Immunol. 7 : 349. 